\title{
Carry-over Effect
}

National Cancer Institute

\section{Source}

National Cancer Institute. Carry-over Effect. NCI Thesaurus. Code C142409.

Therapeutic responses that continue beyond cessation of treatment, and sometimes, beyond a pharmaceutical product's established biological action. 